\title{
Klinik, Diagnostik und Pathophysiologie der transienten globalen Amnesie (TGA)
}

Carolin Hoyer, Kristina Szabo

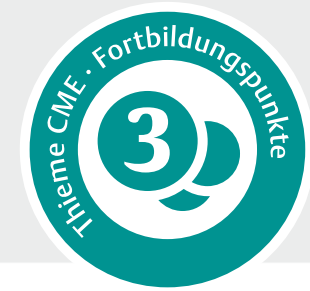

Bei der transienten globalen Amnesie (TGA) handelt es sich um eine vorübergehende, akut auftretende Gedächtnisstörung mit antero- und retrograder Amnesie für die Dauer von bis zu 24 Stunden ohne Bewusstseinsminderung oder weitere neurologische Ausfälle. Typisches Merkmal der TGA sind umschriebene hippokampale Läsionen in der Kernspintomografie, die am zuverlässigsten mit ca. 24 Stunden Latenz zur Episode nachgewiesen werden können.

\section{Historie und Epidemiologie der transienten globalen Amnesie}

Trip Gabriel, ein amerikanischer Journalist, ist 61 Jahre alt, als er am 27. Juni 2015 in einem Kernspintomografen zu sich kommt und sich fragt, wie er dorthin gekommen ist. Kurze Zeit darauf, in seinem Krankenhauszimmer, findet er eine Post-it-Notiz von seiner Frau, auf der steht:

You have a condition called transient global amnesia. It will last HOURS, not DAYS. You're going to be fine. Your MRI scan was clear. You sailed today and drove yourself home. The neurologist says your prognosis is GREAT. You have not had a stroke! There will be no permanent damage [1].

Die transiente globale Amnesie (TGA) ist ein hochinteressantes und noch nicht komplett verstandenes Krankheitsbild der klinischen Neurologie. Eine italienische Studie aus dem Jahr 2014 gibt die jährliche Inzidenz der TGA mit 6,4/100000 Einwohner an [2]. Verschiedene Studien konnten keine einheitliche Geschlechterverteilung nachweisen, wohl aber ein häufigeres Auftreten in der 5. und 6. Lebensdekade [3].

Die klinische Phänomenologie der TGA ist schon seit den 1960er-Jahren im Detail anhand zahlreicher Kasuistiken und Fallserien beschrieben [4,5], allerdings werden immer noch zahlreiche Fallbeispiele veröffentlicht, die die Faszination dieser Störung in der klinischen Neurologie widerspiegeln. Im Jahr 1985 veröffentlichte Caplan die erste klinische Definition des Syndroms im „Handbook of Clinical Neurology“ [4]. Demzufolge müssen die folgenden vier Kriterien erfüllt sein, um die Diagnose einer TGA stellen zu können:
- Der Beginn der TGA muss von einem Beobachter bezeugt sein.

- Die Untersuchung des Patienten muss weitere neurologische Symptome ausschließen, die auf eine andere Ursache der Amnesie deuten könnten.

- Die Amnesie darf nicht von weiteren neurologischen Symptomen begleitet oder gefolgt werden.

- Die Amnesie muss sich zurückbilden.

Die durch Hodges und Warlow im Jahre 1990 veröffentlichte Modifikation der diagnostischen Kriterien (s. Übersicht) besitzt bis heute Gültigkeit und wird im klinischen Alltag angewandt [6].

\section{Merke}

Die TGA ist eine akut einsetzende Gedächtnisstörung und tritt überwiegend bei Menschen jenseits des 50 . Lebensjahres auf.

Ein typisches Beispiel aus dem klinischen Alltag ist in diesem Artikel als fortlaufende Kasuistik beschrieben.
ABKÜRZUNGEN
CCT kraniale Computertomografie
cMRT kraniale Magnetresonanztomografie
CNR Kontrast-zu-Rauschen-Verhältnis
DWI Diffusion-weighted Imaging
LP Lumbalpunktion
MMSE Mini-Mental State Examination
MoCa Montreal Cognitive Assessment
RCVS reversibles zerebrales Vasokonstriktionssyndrom
SNR Signal-zu-Rauschen-Verhältnis
TGA transiente globale Amnesie 


\section{PRAXIS}

Diagnosekriterien einer transienten globalen Amnesie nach Hodges und Warlow

- Die Episode muss beobachtet sein bzw. es müssen Informationen von einem geeigneten Zeugen vorhanden sein, der die Attacke vollständig miterlebt hat.

- Es muss eine eindeutig abzugrenzende anterograde Amnesie vorliegen.

- Fehlen von kognitiven Defiziten außer der Amnesie (z. B. Aphasie etc.), einer Bewusstseinsstörung und von Desorientierung zur Person.

- Keine fokalen neurologischen Ausfälle während und nach TGA.

- Keine klinischen Merkmale, die auf einen epileptischen Anfall hindeuten.

- Kein vorangehendes Trauma oder Epilepsie (insbesondere epileptische Anfälle in den letzten 2 Jahren).

- Dauer mindestens $1 \mathrm{~h}$, Rückbildung innerhalb $24 \mathrm{~h}$.

(nach [6])
Aufgrund des typischen Musters der neuropsychologischen Symptomatik während der TGA hat man schon früh eine zugrundeliegende Dysfunktion des Temporallappens postuliert. Nicht zuletzt aus Läsionsstudien wusste man um die Bedeutung insbesondere des Hippokampus für die langfristige Speicherung von Gedächtnisinhalten, der Gedächtniskonsolidierung: Der als K.C. in die Gedächtnisforschung eingegangene Patient war nach einer schweren Contusio cerebri nie wieder in der Lage, neue Gedächtnisinhalte zu bilden, und erinnerte sich nur an Begebenheiten vor seinem Motorradunfall. In der MRT seines Gehirns hatten sich bilateral schwere kontusionelle Läsionen des Hippocampus gezeigt [7].

Die TGA bietet daher als klinisches Modell einer vorübergehenden hippokampalen Störung die außergewöhnliche Möglichkeit, komplexe neuropsychologische Modelle der Gedächtnisprozesse am Menschen zu untersuchen. Neuropsychologische Untersuchungen in der Akutphase und im Verlauf der TGA weisen darauf hin, dass auch andere - wenn auch subtile - Störungen weiterer neurokognitiver Bereiche wie z. B. der Exekutivfunktion bestehen können [8].

\section{Neuropsychologisches Störungs- profil}

Die TGA ist gekennzeichnet durch eine plötzlich auftretende anterograde Amnesie mit Beeinträchtigung des episodischen Langzeitgedächtnisses. Dies führt dazu, dass sich die Betroffenen keine neuen Informationen länger als ca. 3-5 Minuten merken können und daher wiederholt dieselben Fragen zu ihrer Umgebung und den Begleitumständen stellen, was zum charakteristischen Phänomen des repetitiven Nachfragens führt.

Eine begleitende retrograde Gedächtnisstörung ist häufig weniger schwer und im zeitlichen Ausmaß unterschiedlich stark ausgeprägt. Aber auch diese kann in Einzelfällen sehr prominent sein, so z. B., wenn sich Betroffene nicht an wichtige Ereignisse in der Vergangenheit (z. B. Geburten oder Todesfälle) erinnern können.

Patienten sind während einer TGA zur Person vollständig orientiert und können auch ohne weiteres auf Inhalte aus dem expliziten Langzeitgedächtnis (Autobiografie und Faktenwissen) zugreifen, sodass sie Fragen zur eigenen Person (z. B. das Geburtsdatum) oder (je nach Schweregrad der retrograden Amnesie) beispielsweise zum aktuellen Weltgeschehen richtig beantworten können, jedoch nicht wissen, welcher Wochentag ist oder wie sie an den aktuellen Aufenthaltsort gelangt sind. Patienten sind während der Episode in der Lage, komplexe Handlungen wie beispielsweise Autofahren auszuführen.

\section{Merke}

Das funktionelle Korrelat der bei der TGA zu beobachtenden anterograden Amnesie ist eine vorübergehende hippokampale Störung der Gedächtniskonsolidierung.

\section{Diagnostisches Vorgehen}

Häufig findet sich eine typische (Fremd-)Anamnese mit einer akuten Verwirrtheit und repetitivem Nachfragen. Als kurze neuropsychologische Untersuchung eignet sich z. B. ein 5-Item-Test des verbalen Gedächtnisses. Man fordert den Patienten auf, die genannten Begriffe zu wiederholen und sich zu merken und erfragt diese nach ca. 5-10 Minuten. Während der Akutphase einer TGA können sich Betroffene an die Wörter nicht erinnern, ebenso wenig an den Untersucher, falls er zwischendurch den Untersuchungsraum verlässt.

- Tab. 1 fasst eine kurze neuropsychologische Testung bei V.a. TGA zusammen.

\section{Merke}

Die Amnesie verursacht eine Störung der Orientierung zu Zeit, Ort und Situation, wohingegen die Orientierung zu Person intakt ist.

Die klinisch-neurologische Untersuchung ist ansonsten unauffällig. Physische oder vegetative Symptome wie Angst, Tachykardie, Blutdruckentgleisung, Schwindel oder Kopfschmerzen können eine TGA begleiten, stehen aber erfahrungsgemäß nie im Vordergrund. 
- Tab. 1 Kurze neuropsychologische Testung der transienten globalen Amnesie (TGA).

\begin{tabular}{|c|c|}
\hline $\begin{array}{l}\text { Kognitive } \\
\text { Domäne (adap- } \\
\text { tiert nach Test- } \\
\text { verfahren) }\end{array}$ & Vorgehensweise/Fragen \\
\hline \multirow{16}{*}{$\begin{array}{l}\text { Orientierung zu } \\
\text { Person, Zeit und } \\
\text { Ort (MMSE) }\end{array}$} & Beantworten Sie die folgenden Fragen: \\
\hline & zur Person \\
\hline & - Name? \\
\hline & - Nachname? \\
\hline & - Geburtsdatum? \\
\hline & - Adresse (Straße/Stadt)? \\
\hline & zur Zeit \\
\hline & - Wochentag? \\
\hline & - Genaues Datum (Jahr/Monat/Tag)? \\
\hline & - Uhrzeit? \\
\hline & zum Ort \\
\hline & - Land? \\
\hline & - Bundesland? \\
\hline & - Stadt? \\
\hline & - Genauer Aufenthaltsort? \\
\hline & - Etage? \\
\hline $\begin{array}{l}\text { verbales Lang- } \\
\text { zeitgedächtnis } \\
\text { (MoCA) }\end{array}$ & $\begin{array}{l}\text { Nachsprechen (zweimalig), Merken und } \\
\text { Abrufen (nach 5-10 Minuten) einer } \\
\text { Wortliste: } \\
\text { Gesicht/Samt/Kirche/Tulpe/rot }\end{array}$ \\
\hline \multicolumn{2}{|c|}{$\begin{array}{l}\text { Abkürzungen: } \\
\text { MMSE = Mini-Mental State Examination } \\
\text { MoCA = Montreal Cognitive Assessment }\end{array}$} \\
\hline
\end{tabular}

Bei typischer Präsentation ist keine weitere Zusatzdiagnostik notwendig. Falls die Episode nicht von einem zuverlässigen Zeugen beobachtet wurde oder im Falle von anderen untypischen Aspekten (z. B. neurologische Ausfälle, vorangehendes Trauma, initialer epileptischer Anfall), ist eine weitere Zusatzdiagnostik indiziert:

- kraniale Bildgebung,

- EEG,

- ggf. Doppler-/Duplexsonografie der hirnversorgenden Gefäße,

- ggf. LP.

\section{Differenzialdiagnosen}

Mögliche Differenzialdiagnosen sind in $>$ Tab. 2 aufgelistet.
Cave

Red Flags, die an der Diagnose einer TGA zweifeln

lassen sollten, sind:

- neurologischen Ausfälle,

- Fieber,

- Delir,

- Halluzinationen,

- epileptische Anfälle,

- motorische Automatismen,

- Halluzinationen,

- Bewusstseinsveränderungen.

\section{FALLBEISPIEL}

Herr M., ein 64-jähriger Mann, fuhr seine Frau um 9:00 zur Arbeit. Gegen 11:30 Uhr wurde er von seiner Tochter im Garten angetroffen. Er wirkte ratlos und stellte immer wieder die gleichen Fragen, sodass seine Tochter den Rettungsdienst rief. In der Notaufnahme konnte er keine Angaben über die vorausgegangenen Ereignisse des Tages machen, und es fiel auf, dass er während des Gesprächs keine neuen Informationen behalten konnte. Er fragte immer wieder: „Muss ich jetzt hierbleiben?“, und: „Was stimmt mit mir nicht?".

In der neurologisch/neuropsychologischen Untersuchung war er zur Person voll (5/5), zu Zeit nicht $(0 / 5)$ und zu Ort teilweise (2/5) orientiert. Er zeigte eine schwere Beeinträchtigung des verbalen Kurzzeitgedächtnisses (0/5).

\section{Relevanz der Zusatzdiagnostik}

Seit ersten Fallberichten aus dem Jahr 1998 und größeren Serien seit 2004 ist bekannt, dass bei der TGA mit der diffusionsgewichteten MR-Bildgebung typische Läsionen bevorzugt in der CA1-Region des Hippokampus zu finden sind. Das Besondere am Läsionsnachweis bei der TGA ist, dass diese Läsionen in der Akutphase nicht nachweisbar sind, sondern erst mit mindestens 12-24 Stunden Verzögerung auftreten. Da es sich um jeweils nur sehr kleine Läsionen handelt, spielt eine optimierte Untersuchungstechnik eine wichtige Rolle. Hierbei müssen das Signal-zu-Rauschen- und Kontrast-zu-Rauschen-Verhältnis (s. Infobox „Hintergrundinformation) optimiert sein. Darüber hinaus sollten die bestmöglichen Ebenen zur Hippokampusdarstellung gewählt werden:

- parallel und

- senkrecht zum Verlauf des Hippokampus. 
> Tab.2 Differenzialdiagnosen der transienten globalen Amnesie (TGA).

\begin{tabular}{|c|c|}
\hline Differenzialdiagnose & Bemerkungen \\
\hline $\begin{array}{l}\text { zerebrovaskuläres Ereignis } \\
\text { (insbesondere vertebrobasiläres } \\
\text { Stromgebiet) }\end{array}$ & $\begin{array}{l}\text { Klinik, Symptomatik: } \\
\text { " Bewusstseinsstörung } \\
\text { " Desorientierung auch zur Person } \\
\text { " neurologische Defizite (insbesondere Doppelbilder, Schluckstörungen, Hemianopsie, Sensibi- } \\
\text { litätsstörungen, Paresen, etc.) } \\
\text { Indizierte Zusatzdiagnostik: } \\
\text { " kraniale Bildgebung (cMRT, bei Kontraindikationen cCT) } \\
\text { - Doppler-/Duplexsonografie der hirnversorgenden Gefäße } \\
\text { " EKG-Monitoring/Langzeit-EKG } \\
\text { " Echokardiografie }\end{array}$ \\
\hline Commotio cerebri & $\begin{array}{l}\text { Klinik, Symptomatik: } \\
\text { " (Fremd-)Anamnese hinweisend auf ein vorangehendes Trauma } \\
\text { " Prellmarken } \\
\text { " Bewusstseinsstörung } \\
\text { - Desorientierung auch zur Person } \\
\text { " neurologische Defizite } \\
\text { " Erbrechen } \\
\text { Indizierte Zusatzdiagnostik: } \\
\text { - kraniale Bildgebung (cMRT, cCT) }\end{array}$ \\
\hline epileptische Anfälle & $\begin{array}{l}\text { Klinik, Symptomatik: } \\
\text { " Dauer< } 1 \text { Stunde } \\
\text { " anamnestisch rezidivierende Episoden } \\
\text { " Alter oft < } 40 \text { Jahre } \\
\text { " kein repetitives Nachfragen } \\
\text { Indizierte Zusatzdiagnostik: } \\
\text { " EEG } \\
\text { - kraniale Bildgebung } \\
\text { Insbesondere transiente epileptische Amnesie: } \\
\text { - kürzere Dauer und wiederholtes Auftreten typisch } \\
\text { " häufig mit motorischen Automatismen, Bewusstseinsveränderungen oder Halluzinationen } \\
\text { - Nachweis iktaler Aktivität im EEG }\end{array}$ \\
\hline Intoxikationen & $\begin{array}{l}\text { Klinik, Symptomatik: } \\
\text { " (Fremd-)Anamnese, insbesondere Medikamentenanamnese } \\
\text { - Einnahme von Drogen } \\
\text { " Bewusstseinseintrübung } \\
\text { - Desorientierung auch zur Person } \\
\text { Indizierte Zusatzdiagnostik: } \\
\text { " toxikologisches Screening } \\
\text { - EEG } \\
\text { - ggf. kraniale Bildgebung. }\end{array}$ \\
\hline
\end{tabular}

Auch hat sich die Befundbestätigung in einer zweiten Ebene für die Läsionsdetektion als hilfreich erwiesen.

\section{HINTERGRUNDINFORMATION \\ Optimierte MRT-Untersuchungstechnik}

- höhere Feldstärle

- geringere Schichtdicke

- höhere b-Werte

- Schichtführung parallel zum Hippokampus, ggf. zweite Ebene
Bei entsprechender Berücksichtigung der zeitlichen und technischen Planung der Untersuchung kann man bei bis zu 70\% der TGA-Patienten entsprechende Veränderungen finden [9]. Diese können uni- oder bilateral sein und einzeln oder mehrfach auftreten ( $\triangleright$ Abb. $\mathbf{1}$ ).

\footnotetext{
Merke

Obwohl es klinische Kriterien für die Diagnose TGA gibt, kommt der MRT eine besondere Bedeutung zu, da der Nachweis typischer Läsionen geeignet ist, die Verdachtsdiagnose einer TGA im positiven Sinne zu stützen. Dabei erleichtern optimierte MRT-Sequenzen die Detektion TGA-assoziierter Läsionen des Hippokampus.
} 
Tab. 2 (Fortsetzung)

\begin{tabular}{|c|c|}
\hline Differenzialdiagnose & Bemerkungen \\
\hline Enzephalitis & 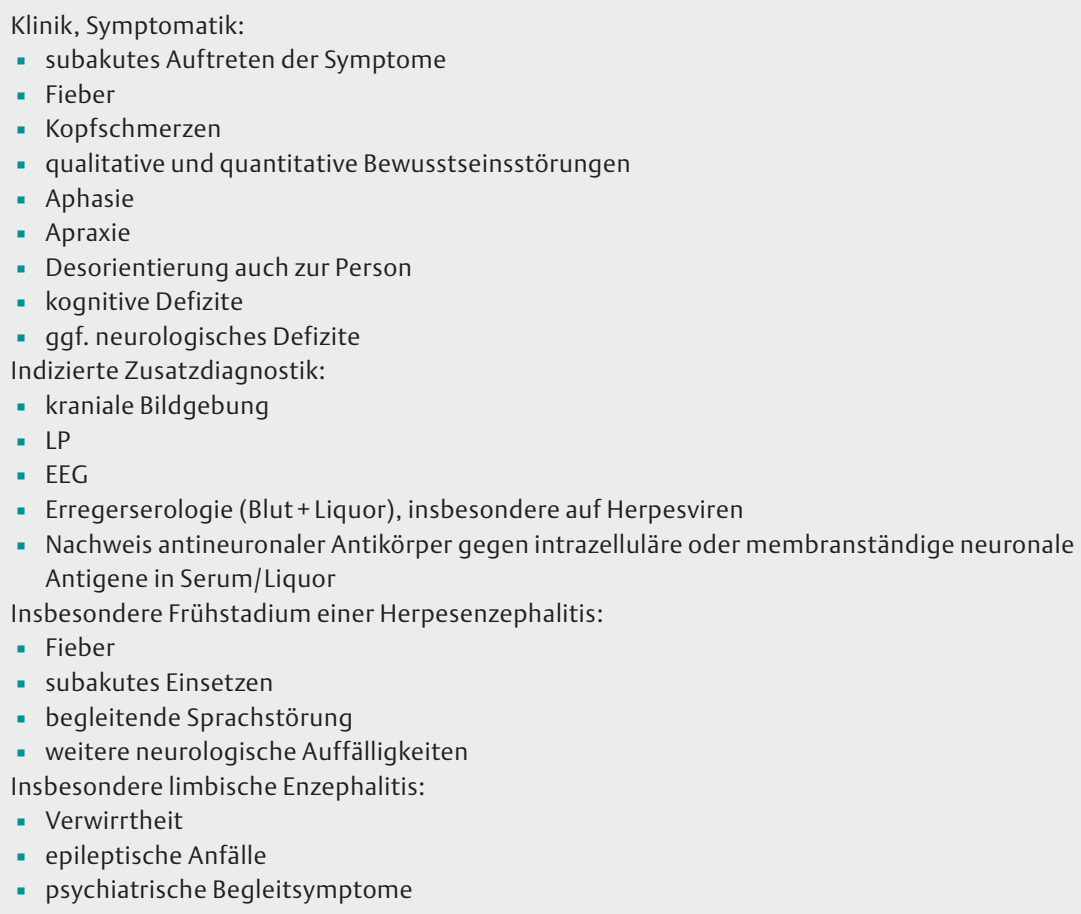 \\
\hline $\begin{array}{l}\text { psychogene/funktionelle Stö- } \\
\text { rung }\end{array}$ & $\begin{array}{l}\text { Klinik, Symptomatik: } \\
\text { " Alter meist< } 40 \text { Jahre } \\
\text { " hauptsächlich retrograde Amnesie } \\
\text { " Desorientierung auch zur Person } \\
\text { - Dauer häufig > } 24 \mathrm{~h} \\
\text { Indizierte Zusatzdiagnostik: } \\
\text { - } \text { nach Ausschluss einer organischen Genese psychiatrische Behandlung }\end{array}$ \\
\hline $\begin{array}{l}\text { Elektrolytveränderungen/Hypo- } \\
\text { glykämie }\end{array}$ & $\begin{array}{l}\text { Klinik, Symptomatik: } \\
\text { - Bewusstseinsstörungen } \\
\text { - Desorientierung auch zur Person } \\
\text { Indizierte Zusatzdiagnostik: } \\
\text { - Blutentnahme/Labor }\end{array}$ \\
\hline
\end{tabular}

Nach wie vor jedoch ist die Entstehung dieser Läsionen ungeklärt-am ehesten entsprechen sie einem Epiphänomen, können jedoch nicht das klinische Korrelat der Gedächtnisbeeinträchtigung vollständig erklären oder gar die Ursache der TGA sein.

EEG-Untersuchungen im Rahmen der TGA sind in der Regel unauffällig oder zeigen unspezifische Veränderungen wie den gelegentlichen Nachweis von Theta-/ Delta-Wellen in den temporalen Ableitungen. Eine ausführliche neuropsychologische Testung kann bei atypischer klinischer Präsentation zur Abgrenzung gegenüber einer demenziellen Erkrankung - insbesondere im Verlauf - sinnvoll sein.

\section{Spontanverlauf und Prognose der transienten globalen Amnesie}

In der Regel bildet sich die Gedächtnisstörung spontan nach 6 - 8 Stunden, definitionsgemäß nach spätestens 24 Stunden zurück. Aus einer sehr aktuellen Analyse stammt die Beobachtung, dass TGA-Episoden weniger als $1 \mathrm{~h}$ andauern können, was wiederum die Relevanz einer bestätigenden Untersuchung unterstreicht [10].

Merke

Nach der Episode bleibt eine amnestische Lücke für die Episode selbst, alle anderen Gedächtnisfunktionen normalisieren sich vollständig. 


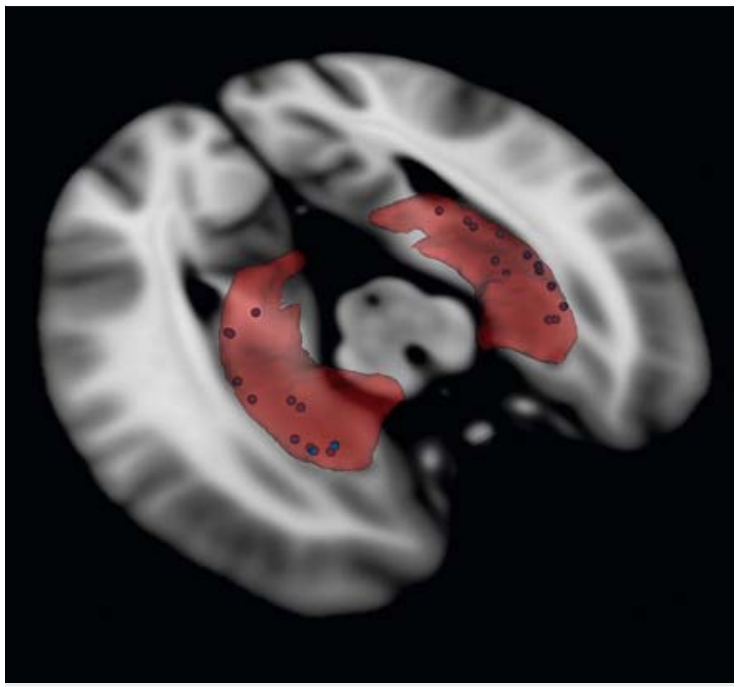

- Abb. 1 3D-Volumetrie des Hippokampus mit schematischer Überlagerung der akuten TGA-Läsionen aus einem Kollektiv von 13 Patienten mit transienter globaler Amnesie (TGA).
Mehrere Studien haben das Wiederauftreten der TGA und die Frage von zerebrovaskulären oder neurokognitiven Folgeerkrankungen in größeren Kollektiven untersucht. Diese Aspekte sind im klinischen Alltag von besonderer Relevanz, da sie zu den häufigsten Fragen und Ängsten von Patienten und Angehörigen gehören. Mehrere aktuelle Untersuchungen geben die Wahrscheinlichkeit wiederholter TGA-Episoden mit 5-8\% an, frühere Studien kommen auf $10-18 \%$ [11, 12]. Allerdings wurde bei Patienten nach TGA - entsprechend den Ergebnissen früherer Studien - kein erhöhtes Risiko für eine zerebrovaskuläre Erkrankung, epileptische Anfälle oder das Auftreten einer kognitiven Störung im Sinne einer Demenz gefunden.

In einer Verlaufsstudie mit 7 Tesla-MRT konnte gezeigt werden, dass die hippokampalen Läsionen keine morphologischen Veränderungen verursachen [13].

\section{FALLBEISPIEL}

Bei Herrn M. wurde eine transiente globale Amnesie (TGA) diagnostiziert. Seine Gedächtnisstörung bildete sich innerhalb von 7 Stunden nach der Einlieferung vollständig zurück. In einer anschließenden neuropsychologischen Testung waren sämtliche Gedächtnisleistungen wieder normal. Allerdings behielt er eine Gedächtnislücke für die Dauer von etwa 8 Stunden.

Sämtliche Untersuchungen waren unauffällig. Lediglich in der am Folgetag durchgeführten Kernspintomografie des Gehirns fanden sich kleine signalangehobene Läsionen im rechten Hippokampus ( $\downarrow$ Abb. 2). Wie wir später erfuhren, hatte sich die TGA am Todestag seines im Vorjahr verstorbenen Bruders ereignet.

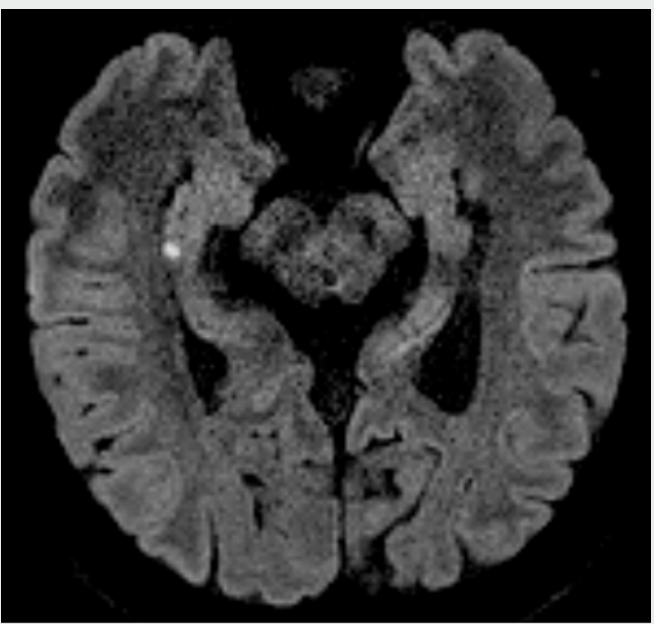

Abb. 2 Kraniale Kernspintomografie: Die parallel zur Längsachse des Hippokampus geplante diffusionsgewichtete (DWI) MR-Aufnahmen zeigen 48 Stunden nach Beginn der TGA-Episode eine punktförmige Läsion im rechten Hippokampus. 
FALLBEISPIEL

An Tag 4 nach der TGA berichtete Herr M.: „Ich kann jetzt rekonstruieren, dass ich für die Zeit zwischen halb 11 Uhr am Vormittag und halb 5 am Nachmittag keine Erinnerung habe. Ich kann mich an Dinge um 9 Uhr morgens noch erinnern. Ich hatte zum Beispiel ausgemacht, dass ich meine Frau zur Arbeit fahre, aber dass ich es tatsächlich gemacht habe, weiß ich nicht mehr. Das erste, woran ich mich an diesem Tag dann wieder erinnern kann, ist, dass mich ein Arzt in mein Zimmer auf Station gebracht hat. Meine Frau war auch dabei. Als sie dann sagte, dass sie jetzt gehen müsse, habe ich ihr gesagt, ,Du bist doch eben erst gekommen', dabei war sie schon seit 5 Stunden da.“

\section{Therapie}

Bei abklingender Symptomatik und diagnostischer Sicherheit ist eine ambulante Führung des Patienten vertretbar, sofern der Patient unter Aufsicht einer Bezugsperson bleibt. Bei anhaltender Symptomatik oder Zweifel an der Diagnose sollte eine stationäre Aufnahme zur Überwachung und weiteren Abklärung erfolgen. Aktuell existieren weder eine spezifische Therapie noch Empfehlungen hinsichtlich einer Prophylaxe-beides erscheint allerdings aufgrund der Gutartigkeit der Störung nicht notwendig.

\section{FAZIT}

\section{Take-Home Message}

Die TGA ist eine vorübergehende und gutartige Störung des Gehirns ohne Folgeerkrankungen. Eine Therapie oder Prophylaxe existiert nicht, ist aber wahrscheinlich auch nicht notwendig.

\section{Trigger und Begleiterkrankungen der transienten globalen Amnesie}

Interessante Fallberichte machen unverändert einen relevanten Anteil an Veröffentlichungen über die TGA aus. Besonders hingewiesen werden soll auf solche TGA-Episoden, die im Rahmen von anderen akuten neurologischen Krankheitsbildern berichtet worden sind. So sind zuletzt mehrere Fälle eines reversiblen zerebralen Vasokonstriktionssyndroms (RCVS) mit begleitender TGA veröffentlicht worden [14]. In einem dieser Fälle erfolgte die Diagnose des RCVS erst, nachdem aufgrund von anhaltenden Kopfschmerzen im
Verlauf eine kernspintomografische Untersuchung des Gehirns veranlasst wurde.

Ebenso finden sich häufige Berichte von dem Anschein nach klassischen TGA-Attacken, im Rahmen deren Abklärung sich unerwartete Diffusionsrestriktionen extrahippokampal zeigen, z. B. im Gyrus cinguli oder im Putamen $[9,15]$. Bislang ist die Relevanz dieser Befunde insbesondere in Abgrenzung zu zerebralen Ischämien nicht geklärt. Eine vaskuläre Genese der hippokampalen Läsionen - im Sinne eines akuten Schlaganfalls - bei fehlenden Hinweisen auf Gefäßpathologien oder Perfusionsstörungen sowie dem Fehlen von TGA-Symptomen im Rahmen von akuten Durchblutungsstörungen des Hippokampus konnte nicht erhärtet werden.

\section{Merke \\ Diese Befunde unterstreichen jedoch die Bedeutung der MRT-Bildgebung zum Ausschluss etwaiger Diffe- renzialdiagnosen oder Begleiterkrankungen.}

Interessanterweise haben bereits frühere anekdotische Arbeiten Auslösesituationen der TGA nahegelegt, die man unter dem Begriff „psychischer und physischer Stress“ zusammenfassen könnte [5]. Neben Kontakt mit kaltem Wasser können große Wut, Freude, Trauer, ein plötzlicher Wetterwechsel sowie körperliche Anstrengung solche Auslöser sein.

Epidemiologische Studien haben in bestimmten TGAKollektiven wiederholt eine höhere Migräneprävalenz zeigen können. Neben der Migräne wurde auch das häufigere Auftreten bestimmter Befunde bei TGA-Patienten beobachtet:

- phobische Persönlichkeitsmerkmale,

- depressive Symptome sowie

- psychiatrische Begleiterkrankungen [9].

Interessante Beobachtungen der letzten Jahre bringen die TGA mit kardialen Befunden, so zum Beispiel mit einem Myokardinfarkt oder mit einer Troponin-Erhöhung, in Verbindung [16]. Ebenso gibt es wiederholte Fallbeschreibungen einer Koinzidenz der TGA mit der möglicherweise ebenfalls stressvermittelten Takotsubo-Kardiomyopathie. Hieraus resultiert die wissenschaftliche Untersuchung möglicher wechselseitiger funktionaler Zusammenhänge zwischen dem Gehirn und dem Herzen. 


\section{Mögliche pathophysiologische Mechanismen der transienten globalen Amnesie}

\author{
Merke \\ Die genaue Ätiologie und Pathophysiologie der TGA \\ ist nicht geklärt.
}

Wissenschaftliche Erkenntnisse deuten auf eine multifaktorielle Genese und auf mögliche Subgruppen mit spezifischen Triggern. Mehrere frühere Arbeiten hatten - basierend auf einer Hypothese von Lewis im Jahre 1998-eine venöse Abflussstörung des Gehirns mit konsekutiver venöser Ischämie im Hippokampus im Rahmen eines Valsalva-Manövers als mögliche Ursache der TGA postuliert [17]. Nach dieser Theorie sollte es durch den erhöhten intrakraniellen Druck zu einem reduzierten venösen Rückstrom mit resultierender intrakranieller venöser Hypertension und Kongestion im Temporallappen kommen. In diese Richtung wiesen Berichte über der TGA vorausgehende Valsalva-ähnlichen Manöver und der Nachweis von inkompetenten Klappen der V. jugularis sowie von venösem Reflux bei TGA-Patienten.

Diese Hypothese der venösen Ischämie wurde durch mehrere negative Veröffentlichungen in den vergangenen Jahren und den fehlenden Nachweis einer hämodynamischen Relevanz venöser Befunde weitgehend entkräftet. Die vaskulär-ischämische Genese der TGA ist unwahrscheinlich. Größere Studien konnten weder eine erhöhte Schlaganfallinzidenz nach TGA noch eine entsprechende Konstellation der Risikofaktoren bei TGA-Patienten finden.

Neben diesen vaskulären Mechanismen werden bezüglich der Pathophysiologie der TGA auch humorale, genauer neuroendokrine, Faktoren diskutiert. Eine wesentliche Rolle hierbei scheint eine Glutamat-vermittelte Hyperexzitabilität zu spielen, die zu einer zytotoxischen Schädigung des Hippokampus, insbesondere der CA1-Neurone, führt. Als ein möglicher Auslöser einer solchen Kaskade wurden schon früh Migräne-ähnliche Prozesse - insbesondere die „spreading Depression“ vermutet $[18,19]$, da epidemiologische Studien in bestimmten TGA-Kollektiven wiederholt eine häufigere Migräneanamnese berichteten. Der Nachweis eines solchen Phänomens bei der TGA konnte bislang aber nicht erbracht werden.

Aus den Beobachtungen zu Auslösern und psychischen Begleiterkrankungen resultiert ein weiteres interessantes Erklärungsmodell der TGA, dem eine Stress- und Stresshormon-vermittelte Kaskade und eine hieraus resultierende vorübergehende transiente Störung der Energieversorgung im Hippokampus zugrunde liegt.
Erste Studien fanden Hinweise für eine veränderte Stressempfindlichkeit der neuroendokrinen Achse bei TGA-Patienten [20].

\section{FAZIT}

Take-Home Message

Obwohl in der Erforschung der Ursachen der TGA in den vergangenen Jahren unterschiedliche pathophysiologische Ansätze verfolgt worden sind, bleibt die Ätiologie weiterhin ungeklärt.

\section{Schlussfolgerung}

Die TGA ist einer der interessantesten Gedächtnisstörungen der klinischen Neurologie. Zahlreiche Aspekte wie pathophysiologische Relevanz der Auslöser, die ausgestanzte hippokampale Symptomatik und die Befunde in der MRT-Bildgebung bleiben trotz der Verfolgung unterschiedlicher pathophysiologischer Ansätze unverstanden.

\section{KERNAUSSAGEN}

- Die transiente globale Amnesie (TGA) ist gekennzeichnet durch eine plötzlich auftretende anterograde Amnesie, die dazu führt, dass sich die Betroffenen vorübergehend keine neuen Informationen merken können und daher wiederholt dieselben Fragen zu ihrer Umgebung stellen.

- Eine begleitende retrograde Gedächtnisstörung ist häufig weniger schwer ausgeprägt.

- Bei typischer und beobachteter Symptomatik sowie bei typischer Fremdanamnese kann die Diagnose in vielen Fällen klinisch durch einen erfahrenen Arzt gestellt werden.

- Der MRT kommt eine besondere Bedeutung zu, da der Nachweis typischer Läsionen die Verdachtsdiagnose einer TGA im positiven Sinne stützen kann.

- Die TGA stellt kein Risiko für eine spätere neurovaskuläre oder neurokognitive Erkrankung dar.

- Trotz vielversprechender Ansätze und Hypothesen ist die Ätiologie der TGA ungeklärt. 


\section{Interessenkonflikt}

Erklärung zu finanziellen Interessen

Forschungsförderung erhalten: nein; Honorar/geldwerten Vorteil für Referententätigkeit erhalten: nein; Bezahlter Berater/interner Schulungsreferent/Gehaltsempfänger: nein; Patent/Geschäftsanteile/Aktien (Autor/Partner, Ehepartner, Kinder) an Firma (Nicht-Sponsor der Veranstaltung): nein; Patent/Geschäftsanteile/Aktien (Autor/Partner, Ehepartner, Kinder) an Firma (Sponsor der Veranstaltung): nein.

Erklärung zu nichtfinanziellen Interessen

Die Autorinnen/Autoren geben an, dass kein Interessenkonflikt besteht.

\section{Autorinnen/Autoren}

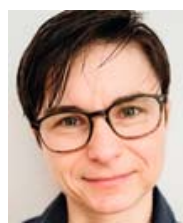

\section{Carolin Hoyer}

Dr. med. 2001-2008 Medizinstudium an der Universität zu Köln, 2008-2014 Facharztweiterbildung Psychiatrie, 2015-2019 Facharztweiterbildung Neurologie. Schwerpunkt: neurologische Notfallmedizin.

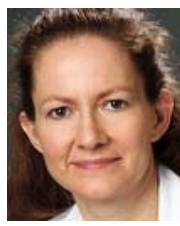

\section{Kristina Szabo}

Prof. Dr. med. 1990-1997 Medizinstudium an der Ruprecht-Karls-Universität Heidelberg, 1998-2004 Facharztweiterbildung Neurologie. Seit 2013 Leitende Oberärztin der Neurologischen Klinik der Medizinischen Fakultät Mannheim der Universität Heidelberg.

Schwerpunkte: neurologische MR-Bildgebung, Diagnostik und Therapie des akuten Schlaganfalls.

\section{Korrespondenzadresse}

\section{Prof. Dr. Kristina Szabo}

Universitätsmedizin Mannheim

Medizinische Fakultät Mannheim der Universität Heidelberg Neurologische Klinik

Theodor-Kutzer-Ufer 1-3

68167 Mannheim

kristina.szabo@umm.de

Wissenschaftlich verantwortlich gemäß

Zertifizierungsbestimmungen

Wissenschaftlich verantwortlich gemäß Zertifizierungsbestimmungen für diesen Beitrag ist Prof. Dr. med. Kristina Szabo, Mannheim.
Literatur

[1] Gabriel T. The day that went missing: a first-person account of transient global amnesia. Cogn Behav Neurol 2017; 30: 1-4. doi:10.1097/WNN.0000000000000115

[2] Brigo F, Lochner P, Tezzon F et al. Incidence of transient global amnesia in Merano, province of Bolzano, Italy. Acta Neurol Belg 2014; 114: 293-296. doi:10.1007/s13760-0140281-3

[3] Lin KH, Chen YT, Fuh JL et al. Migraine is associated with a higher risk of transient global amnesia: a nationwide cohort study. Eur J Neurol 2014; 21: 718-724. doi:10.1111/ ene. 12346

[4] Caplan LR. Transient global amnesia. In: Vinken PJ, Bruyn GW, Klawans HL. Handbook of clinical neurology. Amsterdam: Elsevier; 1985: 205-218

[5] Fisher CM, Adams RD. Transient global amnesia. Acta Neurol Scand 1964; 40: 1-83

[6] Hodges JR, Warlow CP. Syndromes of transient amnesia: towards a classification. A study of 153 cases. J Neurol Neurosurg Psychiat 1990; 53: 834-843. doi:10.1136/ jnnp.53.10.834

[7] Rosenbaum RS, Kohler S, Schacter DL et al. The case of K.C.: contributions of a memory-impaired person to memory theory. Neuropsychologia 2005; 43: 989-1021. doi:10.1016/j.neuropsychologia.2004.10.007

[8] Jager $\mathrm{T}$, Bazner $\mathrm{H}$, Kliegel $\mathrm{M}$ et al. The transience and nature of cognitive impairments in transient global amnesia: a meta-analysis. J Clin Exp Neuropsychol 2009; 31: 819doi:10.1080/13803390801955193

[9] Szabo K, Hoyer C, Caplan LR et al. Diffusion-weighted MRI in transient global amnesia and its diagnostic implications. Neurology 2020: doi:10.1212/WNL.0000000000009783

[10] Romoli M, Tuna MA, Li L et al. Time trends, frequency, characteristics and prognosis of short-duration transient global amnesia. Eur J Neurol 2020; 27: 887-893. doi:10.1111/ ene. 14163

[11] Arena JE, Brown RD, Mandrekar J et al. Long-term outcome in patients with transient global amnesia: a population-based study. Mayo Clin Proc 2017; 92: 399-405. doi:10.1016/ j.mayocp.2016.11.015

[12] Alessandro L, Calandri IL, Suarez MF et al. Transient global amnesia: clinical features and prognostic factors suggesting recurrence. Arq Neuropsiquiatr 2019; 77: 3-9. doi:10.1590/ 0004-282X20180157

[13] Paech D, Kuder TA, Rossmanith $C$ et al. What remains after transient global amnesia (TCA)? An ultra-high field $7 \mathrm{~T}$ magnetic resonance imaging study of the hippocampus Eur J Neurol 2020; 27: 406-409. doi:10.1111/ene.14099

[14] Boitet R, Gaillard N, Bendiab E et al. Concomitant reversible cerebral vasoconstriction syndrome and transient global amnesia. J Neurol 2020; 267: 390-394. doi:10.1007| s00415-019-09594-5

[15] Yoshida K. A Case of transient global amnesia with small left putamen infarction. J Stroke Cerebrovasc Dis 2017; 26: e27-e28. doi:10.1016/j.jstrokecerebrovasdis.2016.10.028 
[16] Eisele P, Baumann S, Noor L et al. Interaction between the heart and the brain in transient global amnesia. J Neurol 2019; 266: 3048-3057. doi:10.1007/s00415-019-09529-0

[17] Lewis SL. Aetiology of transient global amnesia. Lancet 1998; 352: 397-399. doi:S0140-6736(98)01442-1

[18] Crowell GF, Stump DA, Biller J et al. The transient global amnesia-migraine connection. Arch Neurol 1984; 41: $75-$ 79

[19] Bartsch T, Deuschl G. Transient global amnesia: functional anatomy and clinical implications. Lancet Neurol 2010; 9: 205-214. doi:10.1016/S1474-4422(09)70344-8
[20] Griebe M, Ebert A, Nees F et al. Enhanced cortisol secretion in acute transient global amnesia. Psychoneuroendocrinology 2019; 99: 72-79. doi:10.1016/j.psyneuen.2018.08.033

\section{Bibliografie}

DOI https://doi.org/10.1055/a-0948-7329

Neurologie up2date 2020; 03: 259-270

(c) Georg Thieme Verlag KG Stuttgart · New York ISSN 2511-3453 


\section{Punkte sammeln auf CME.thieme.de}

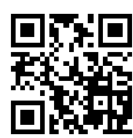

Diese Fortbildungseinheit ist in der Regel 12 Monate online für die Teilnahme verfügbar.

Den genauen Einsendeschluss finden Sie unter https://cme.thieme.de/CXDDF37.

Sollten Sie Fragen zur Online-Teilnahme haben, finden Sie unter https://cme.thieme.de/hilfe

eine ausführliche Anleitung. Wir wünschen viel Erfolg beim Beantworten

der Fragen!

Unter https://eref.thieme.de/CXDDF37 oder über den QR-Code kommen Sie direkt zur Startseite des Wissenstests.

VNR 2760512020158724647

\section{Frage 1}

Wie lange dauert eine transiente globale Amnesie (TGA) gem. den Diagnosekriterien von Hodges und Warlow?
A $\quad 0-1 \mathrm{~h}$
B $1-2 \mathrm{~h}$
C $1-12 \mathrm{~h}$
D $1-24 h$
E $>24 \mathrm{~h}$ mit langsam regredientem Verlauf

\section{Frage 2}

Das führende neuropsychologische Korrelat der Gedächtnisstörung bei der transienten globalen Amnesie ist eine ...
A semantische Amnesie.
$B$ retrograde Amnesie.
C globale Aphasie.
D anterograde und retrograde Amnesie.
E transiente Apraxie.

\section{Frage 3}

Welche Aussage im Rahmen einer Patientenaufklärung über die transiente globale Amnesie (TGA) ist nicht korrekt?

A Die Ursache der TGA ist nicht geklärt.

B Das Risiko einer erneuten Episode ist gering.

C Es gibt keinen Hinweis dafür, dass nach einer TGA das Schlaganfallrisiko erhöht ist.

D Die TGA kann in Einzelfällen das erste Symptom einer Demenz sein.

E Patienten, die eine TGA erleiden, sind häufig jenseits des 50. Lebensjahres.

\section{Frage 4}

Im Falle einer vermuteten transienten globalen Amnesie kann welche Zusatzuntersuchung einen unterstützenden Befund liefern?
A Computertomografie des Gehirns
B Elektroenzephalografie
C Liquorpunktion
D Kernspintomografie des Gehirns
E evozierte Potenziale

\section{Frage 5}

Welcher klinische Befund sollten den Untersucher an der Diagnose einer transienten globalen Amnesie zweifeln lassen?
A Einweisungsdiagnose „akute Verwirrtheit“
B repetitives Nachfragen
C desorientiert zu Zeit
D Vigilanzminderung
E fehlendes fokal-neurologisches Defizit

\section{Frage 6}

Einer der folgenden Punkte gehört nicht zu den Diagnosekriterien der transienten globalen Amnesie nach Hodges and Warlow. Welcher?

A Die Episode muss von einem zuverlässigen Zeugen beobachtet worden sein.

B Es muss eine eindeutige anterograde Amnesie bestehen.

C Beim betroffenen Patienten darf keine Epilepsie bekannt sein.

D Der Patient muss zu Person orientiert sein.

E Die Diagnosestellung muss durch einen Neurologen erfolgen. 


\section{Punkte sammeln auf CME.thieme.de}

Fortsetzung ...

\section{Frage 7}

Welche Aussage zu Auslösern oder Begleiterkrankungen einer transienten globalen Amnesie (TGA) trifft nicht zu?

A Bei Patienten mit der Diagnose einer Schizophrenie konnte ein gehäuftes Auftreten von transienten globalen Amnesien nachgewiesen werden.

B Einer TGA kann eine psychische Belastung vorausgehen.

C Eine TGA kann im Rahmen anderer neurologischer Krankheitsbilder auftreten.

D Epidemiologische Untersuchungen weisen darauf hin, dass TGA-Patienten häufig auch an einer Migräne leiden.

E Einer TGA kann eine körperliche Belastung vorausgehen.

\section{Frage 8}

Wodurch ist der Spontanverlauf der transienten globalen Amnesie (TGA) gekennzeichnet?

A Motorische Automatismen sind hinweisend auf eine TGA.

B Die Gedächtnisstörung im Rahmen der TGA-Episode hält in der Regel 2-3 Wochen an.

C Häufig erholt sich die Gedächtnisfunktion nach einer TGA nicht mehr.

D Die TGA hinterlässt immer eine amnestische Lücke für die Dauer der Störung.

E Viele Patienten leiden nach einer TGA an einer dauerhaften Orientierungsstörung.

\section{Frage 9}

Welche Aussage zum Befund der hippokampalen Läsionen in den diffusionsgewichteten MRT-Sequenzen bei Patienten mit transienter globaler Amnesie trifft zu?

A Punktförmige DWI-Läsionen finden sich immer beidseits im Hippokampus.

B Die hippokampalen Läsionen führen zu chronischen Substanzdefekten im Hippokampus.

C Optimierte MRT-Sequenzen erleichtern die Detektion TGA-assoziierter Läsionen des Hippokampus.

D Die MRT-Untersuchung zum Nachweis hippokampaler Läsionen sollte frühzeitig nach Präsentation des Patienten durchgeführt werden.

E Das Fehlen von hippokampalen Läsionen in der MRT-Bildgebung schließt eine TGA aus.

\section{Frage 10}

Zur Entstehung der transienten globalen Amnesie (TGA) wurden in der Vergangenheit verschiedene Hypothesen postuliert. Welche gehört nicht dazu?

A Stress und Stresshormon-vermittelte Veränderungen könnten eine vorübergehende Störung der Energieversorgung im Hippokampus verursachen.

B Ein erhöhter intrakranieller Druck könnte zu einem reduzierten venösen Rückstrom mit resultierender intrakranieller venöser Hypertension führen.

C Die TGA könnte durch einen Vitamin-D-Mangel verursacht sein.

D Aufgrund der zu beobachtenden hippokampalen DWILäsionen könnte der TGA eine vaskuläre Erkrankung zugrunde liegen.

E Eine zytotoxische Schädigung des Hippokampus im Rahmen einer Glutamat-vermittelten Hyperexzitabilität könnte eine Rolle spielen. 